\title{
Photovoltaic Array Reconfiguration Strategy for Maximization of Energy Production
}

\author{
Paula dos Santos Vicente, Tales Cleber Pimenta, and Enio Roberto Ribeiro
}

Universidade Federal de Itajubá, UNIFEI, Avenida BPS 1303, 37500-903 Itajubá, MG, Brazil

Correspondence should be addressed to Paula dos Santos Vicente; paulasantos@unifei.edu.br

Received 28 November 2014; Revised 3 February 2015; Accepted 3 February 2015

Academic Editor: Emilio Bueno

Copyright (C) 2015 Paula dos Santos Vicente et al. This is an open access article distributed under the Creative Commons Attribution License, which permits unrestricted use, distribution, and reproduction in any medium, provided the original work is properly cited.

\begin{abstract}
This paper presents a dynamic reconfiguration method for electrical connections in a Series-Parallel connected photovoltaic array under partial shading conditions. It is desirable to extract the maximum energy from the array, but it does not occur in situations where the modules have different points of operation caused by shading. The proposed method is then characterized by the maintenance of the PV array dimensions, that is; no module is removed or added to the array. Furthermore, the control algorithm is based on the Rough Sets Theory, which allows the fast and efficient implementation of a control system, comprising rules that identify the system optimal configuration.
\end{abstract}

\section{Introduction}

The use of photovoltaic (PV) systems has increased significantly in recent years as a viable alternative to conventional methods of generating electricity. The reduction of transmission and distribution losses and the possibility of injecting energy into the utility power grid are also attractive factors for their utilization.

The efficiency of PV systems is deeply influenced by weather conditions. In many situations, the PV panel can be illuminated in a nonuniform way due to shading caused by clouds, trees, neighbor buildings, and even shadows caused by the modules in the array.

Shading is a common phenomenon that causes severe degradation to the extraction of energy in PV systems [1]. In a partially shaded array, the output power may be less than the sum of the individual output powers of all modules. The shading of a single module is able to decrease the resulting current and consequently, the overall output power is reduced.

There are several proposals to reduce the degradation of energy in partially shaded systems [2-7]. A common solution is to connect bypass diodes. This connection prevents the existence of rows with low output voltages, which behave as a load to all other parallel connected rows [2].
A different approach lies in systems with microinverters, where each module of the PV array is connected to an inverter with a dedicated MPPT (maximum power point tracking) system [3]. Although this approach offers very good results, since each PV module works at the optimum operating point, its high cost is a drawback.

Recent studies suggest the reconfiguration of the PV array electrical connections. In the reconfiguration system, the PV modules are rearranged dynamically, in order to maximize the output power [4-7].

The reconfiguration method depends on the type of connection between the modules. One of the most common topologies is the Series-Parallel (SP) connection. The $\mathrm{PV}$ modules in this case are connected in series and the resulting rows are connected in parallel. In an SP matrix, the reconfiguration process is based on the shaded modules combination, where modules with similar irradiance levels must be connected in series and the resulting rows are connected in parallel.

Patnaik et al. [4] propose a reconfiguration method in which the modules of the SP array are characterized by two levels: fully illuminated and fully dark. The fully dark modules are removed from the combination, while the fully illuminated modules are rearranged. 
A similar work [5] classifies the PV array modules into three different levels: dark, gray, and bright. The dark modules are removed from the array and the algorithm defines the position of gray and bright modules.

The removal of shaded modules $[4,5]$ may produce asymmetric arrangements. In these situations, the PV array columns have different voltage levels. To solve this problem, static converters can be connected to the disabled rows, thus modifying its voltage levels $[6,7]$.

The aforementioned reconfiguration systems [4-7] have a common feature: the removal of the worst case modules from the array. Once again, the use of static power converters is a possible solution $[6,7]$, but with high cost and complexity of control algorithms.

This paper presents an alternative method for dynamic reconfiguration of the connections in a shaded PV array. The proposed method is characterized by the maintenance of the PV array size; that is, no module is removed or added. Another prominent advantage addressed to the proposal is the use of Rough Sets Theory (RST), which can lead to a fast and efficient algorithm of reconfiguration.

\section{Reconfiguration Method of PV Modules}

2.1. Shading Effect on PV System Efficiency. The performance of PV systems is influenced by irradiance and temperature. Therefore, environmental conditions are crucial for power generation.

A common effect in PV systems is the shading, which can be partial or full, consequently reducing the output power of the PV system. The reduction depends on other factors such as the type of arrangement, shade position, and intensity of shading.

The work in [8] shows, through simulation and experimental tests, that PV arrays with SP interconnection have the best results when shaded modules are grouped. Thus, a significant amount of energy can be recovered, which is not typically extracted when the shaded modules are distributed in the PV array.

2.2. Reconfiguration Method. The reconfiguration method proposed in this paper aims to rearrange the PV array, clustering the shaded modules in the least number of PV array columns.

The rearrangement of shaded modules seems to be a simple and intuitive process. However, depending on the number of modules in the array, this can be a complex task.

In an array with $n$ modules, shading may occur in $0,1,2, \ldots$, or $n$ shaded modules simultaneously. Equation (1) defines the number of different shading possibilities, $S_{P}$ :

$$
S_{P}=2^{n} \text {. }
$$

It is possible to change the position of the modules in an SP array with different ways and $P$ possible configurations, according to simple permutation:

$$
P=n !,
$$

where $n$ is the number of array modules.
In a permutation, the order of the elements is relevant; for example, sequence $\mathrm{ABC}$ is different from sequence $\mathrm{CBA}$. However, from the electrical point of view, the order, in which the PV modules are connected in a series row, does not affect the overall result.

From $P$ possible configurations calculated with (2), only a few ones can produce different results in the system output power. The number of interest settings, $I_{T}$, can be obtained by the combination described in (3). This is related to the symmetry of the PV array (number of rows and columns) [9]:

$$
I_{T}=\frac{(N \cdot M) !}{(N !)^{M} \cdot M !}
$$

where $N$ is the number of rows and $M$ is the number of columns of the PV array.

Among all configurations of interest, one is the most suitable for a given shading profile. This is defined by the amount and position of shaded modules.

The identification of the PV module condition is obtained from parameter $S$, which classifies the module as shaded or unshaded according to (4). This classification can be made through current and voltage measurements:

$$
\begin{gathered}
S=1 \longrightarrow \text { shaded } \\
S=0 \longrightarrow \text { unshaded } .
\end{gathered}
$$

The number of shaded modules is directly related to the possibility of reconfiguration. In an $N$ by $M$ PV array, reconfiguration is feasible in situations where the number of shaded modules, $S_{M}$, is contained in the set defined by

$$
S_{M}=\left\{x \mid x \in \mathbb{N},(\exists y)(\exists z)\left(\begin{array}{c}
y \in\{M\}, z \in\{N\} \\
2 \leq x \leq(z \cdot y-z)
\end{array}\right)\right\} .
$$

Equation (5) defines the set of natural numbers between 2 and the limit $(z \cdot y-z)$, where $z$ and $y$ are numbers contained in the unit sets defined by $N$ and $M$, respectively.

It is important to notice that there are shapes of shade where nothing can be done. In other words, the rearrangement does not cause the output power to be modified. However, in situations where reconfiguration is suitable, at least one configuration may improve the output power. Thereby, the control system is responsible for identifying the best configuration.

\section{Rough Sets Theory (RST)}

The RST is an extension of the set theory, which focuses on the extraction of intrinsic knowledge from a dataset and the creation of rules that summarize the knowledge in this set. It was originally developed by Pawlak at the beginning of the 1980s [10]. However, RST has become quite popular only in the 1990 s due to the increasing of processing capacity and the widespread use of personal computers.

In order to handle the information, data must be organized in a table called information system (IS). IS is a syntactic representation of knowledge of the objects set, represented by the ordered pair $R=(U, A)$. $U$ is a nonempty finite set of 
objects, named Universe, and $A$ is a nonempty finite set of conditional attributes or conditions, such that $U \rightarrow V a$ for $a \in A$. Set $V a$ is defined as the set with $a$ values [11].

RST is based on the indiscernibility, which is an equivalence relationship between objects, given a subset of attributes, which allows the partition of universe set $U$. The general definition is as follows.

Given the information system $R=(U, A)$, then, for any subset of attributes $B \subseteq A$, there is an equivalence relation $\operatorname{IND}(B)$, defined by

$$
\operatorname{IND}(B)=\left\{\left(x, x^{\prime}\right) \in U \mid \forall a \in V, a(x)=a\left(x^{\prime}\right)\right\} .
$$

$\operatorname{IND}(B)$ is the $B$-indiscernibility relationship. If $\left(x, x^{\prime}\right) \epsilon$ $\operatorname{IND}(B)$, then the objects $x$ and $x^{\prime}$ are indiscernible or equal, considering the attributes subset.

The equivalence class of the relation determined by $x$ objects, according to $\operatorname{IND}(B)$, is denoted by $[x] B$.

Based on indiscernibility, it is possible to reduce the extra attributes, obtaining data reduction. A reduction is a subset $B \subseteq A$, where the equivalence classes are preserved by the set of all attributes $\operatorname{IND}(A)$. Thus, for a subset of attributes $B \subseteq A$, it seems that $\operatorname{IND}(B)=\operatorname{IND}(A)$, as $B$ is the reduction of $A$ or $\operatorname{RED}(B)$.

The reduction can be a difficult task depending on the amount of data. It is suitable, therefore, to submit such tables to reduction and simplification procedures. In the simplification process, the unnecessary attributes, duplicated lines, and superfluous values are eliminated. This creates simpler rules that represent the knowledge base of the system, in the same way as the original data.

The manual development of a knowledge base using rough sets is an exhaustive task. Considering this issue, computer programs were developed specifically for this type of application. ROSETTA [12] is one of the most popular approaches, where it is possible to upload a table with system information and choose the method for the creation of classes and reducts. At the end of the process, the system rules are created.

Due to the aforementioned characteristics, RST has been chosen for the implementation of the automatic reconfiguration system.

\section{Reconfiguration System Project}

In order to illustrate the methodology and operation of the proposed reconfiguration system, three photovoltaic arrays are taken as examples.

4.1. $2 \times 2$ Matrix. A $2 \times 2$ matrix (Figure 1) is the smallest SP arrangement in which the reconfiguration technique can be applied. There are a reduced number of shapes of shade where the reconfiguration produces energy improvement. However, this system can be used to evaluate the characteristics and effectiveness of the proposed approach.

According to expression (2) it is possible to obtain 24 combinations for a $2 \times 2$ photovoltaic matrix. Nevertheless, most arrangements are electrically equivalents; that is, they provide the same result.

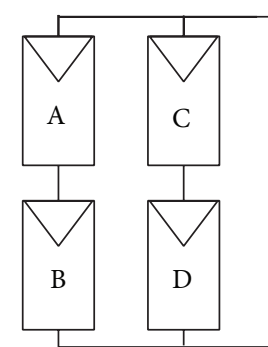

FIGURE 1: CF.01-the $2 \times 2$ SP matrix reference configuration.
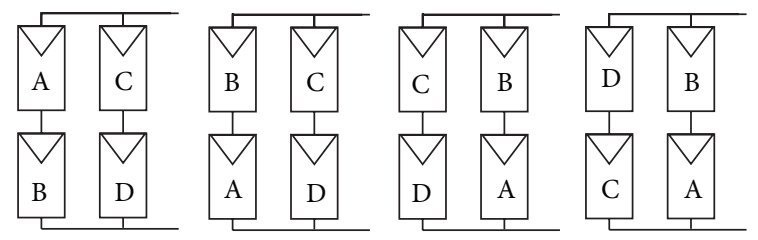

FIgURE 2: Equivalent connections in a $2 \times 2$ matrix.

The arrangements shown in Figure 2 provide the same electrical result. In the four matrices presented, modules $\mathrm{A}$ and $\mathrm{B}$ are in series, $\mathrm{C}$ and $\mathrm{D}$ are also in series, and the two sets are connected in parallel.

According to (3), there are three settings of interest. Among the 24 arrangements of a $2 \times 2$ matrix, 3 configurations do not have equivalencies. Those configurations can be found through combinational analysis and are shown in Figure 3.

According to (1), the number of different possible shadings, in a four modules array, is given by $2^{4}=16$.

Among 16 possible shadings, there are situations where, according to the shapes of shade, the module reconfiguration is relevant. For the $2 \times 2$ array, the unitary set, obtained with (5), is $M_{S}=$ [2]. It means that reconfiguration is performed only in situations where the number of shaded modules is equal to two. If reconfiguration is not suitable, the array will remain in the configuration CF.01, the reference configuration (Figure 1).

The data for the system with four modules are shown in Table 1 . The 16 combinations of shading that can occur in a $2 \times 2$ matrix are represented by the set universe U. A, B, C, and $\mathrm{D}$ represent the conditions attribute (shaded or unshaded) and $\mathrm{CF}$ is the decision attribute, which indicates the optimum configuration.

The decision attribute $\mathrm{CF}$ is obtained from a routine that relates the condition attributes to the reconfiguration criteria defined in Section 2.

Each line of Table 1 represents one of the shading situations that can occur on a $2 \times 2$ matrix and which is the most suitable configuration for that condition.

By using the RST it is possible to obtain the essence of the information and to identify the standard in the data. The data in Table 1 can be reduced with ROSETTA software, which generates eight rules.

(1) $\mathrm{A}(0) \mathrm{AND} \mathrm{B}(0) \rightarrow \mathrm{CF}(01)$

(2) $\mathrm{A}(1) \mathrm{AND} \mathrm{B}(1) \rightarrow \mathrm{CF}(01)$ 


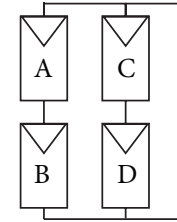

CF.01

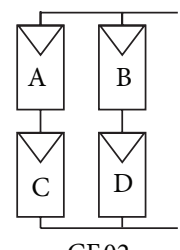

CF.02

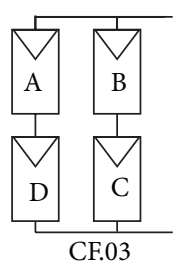

FIgURE 3: Settings of interest in a $2 \times 2$ matrix.

TABLE 1: Information System of a $2 \times 2$ matrix.

\begin{tabular}{llllll}
\hline U & A & B & C & D & CF \\
\hline $\mathbf{1}$ & 0 & 0 & 0 & 0 & CF.01 \\
$\mathbf{2}$ & 0 & 0 & 0 & 1 & CF.01 \\
$\mathbf{3}$ & 0 & 0 & 1 & 0 & CF.01 \\
$\mathbf{4}$ & 0 & 0 & 1 & 1 & CF.01 \\
$\mathbf{5}$ & 0 & 1 & 0 & 0 & CF.01 \\
$\mathbf{6}$ & 0 & 1 & 0 & 1 & CF.02 \\
$\mathbf{7}$ & 0 & 1 & 1 & 0 & CF.03 \\
$\mathbf{8}$ & 1 & 1 & 1 & 1 & CF.01 \\
$\mathbf{9}$ & 1 & 0 & 0 & 0 & CF.01 \\
$\mathbf{1 0}$ & 1 & 0 & 0 & 1 & CF.03 \\
$\mathbf{1 1}$ & 1 & 0 & 1 & 0 & CF.02 \\
$\mathbf{1 2}$ & 1 & 0 & 1 & 1 & CF.01 \\
$\mathbf{1 3}$ & 1 & 1 & 0 & 0 & CF.01 \\
$\mathbf{1 4}$ & 1 & 1 & 0 & 1 & CF.01 \\
$\mathbf{1 5}$ & 1 & 1 & 1 & 0 & CF.01 \\
$\mathbf{1 6}$ & 1 & 1 & 1 & 1 & CF.01 \\
\hline
\end{tabular}

(3) $\mathrm{C}(0)$ AND D(0) $\rightarrow \mathrm{CF}(01)$

(4) $\mathrm{C}(1)$ AND D(1) $\rightarrow \mathrm{CF}(01)$

(5) A(1) AND B(0) AND C(1) AND D(0) $\rightarrow$ CF(02)

(6) A(0) AND B(1) AND C(0) AND D(1) $\rightarrow$ CF(02)

(7) A(1) AND B(0) AND C(0) AND D(1) $\rightarrow \mathrm{CF}(03)$

(8) A(0) AND B(1) AND C(1) AND D(0) $\rightarrow$ CF(03)

The obtained rules express the logic relationship among the condition attributes (A, B, C, and D) and the decision attribute $(\mathrm{CF})$. After combining the eight rules, one can get the three rules listed next. Each rule is related to one of the configurations presented in Figure 3.

(1) $(\mathrm{A}=\mathrm{B})$ OR $(\mathrm{C}=\mathrm{D}) \rightarrow$ CF.01

(2) $(\mathrm{A}=\mathrm{C})$ AND $(\mathrm{B}=\mathrm{D})$ AND $(\mathrm{A} \neq \mathrm{B}) \rightarrow$ CF.02

(3) $(\mathrm{A}=\mathrm{D})$ AND $(\mathrm{B}=\mathrm{C})$ AND $(\mathrm{A} \neq \mathrm{B}) \rightarrow \mathrm{CF} .03$

With these three rules, it is possible to create an algorithm that defines the best configuration for the photovoltaic array under partial shading conditions. Just one of the rules is enabled at a time and the corresponding configuration must be activated so that the system can have the best energy configuration.
TABLE 2: Part of the Information System of a $3 \times 2$ matrix.

\begin{tabular}{lcccccccc}
\hline $\mathrm{U}$ & $\mathrm{A}$ & $\mathrm{B}$ & $\mathrm{C}$ & $\mathrm{D}$ & $\mathrm{E}$ & $\mathrm{F}$ & $\mathrm{Q}$ & $\mathrm{CF}$ \\
\hline $\mathbf{1}$ & 0 & 0 & 0 & 0 & 0 & 0 & 0 & CF.01 \\
$\mathbf{2}$ & 0 & 0 & 0 & 0 & 0 & 1 & 1 & CF.01 \\
$\mathbf{3}$ & 0 & 0 & 0 & 0 & 1 & 0 & 1 & CF.01 \\
$\mathbf{4}$ & 0 & 0 & 0 & 0 & 1 & 1 & 2 & CF.01 or CF.02 or CF.05 or CF.10 \\
$\mathbf{5}$ & 0 & 0 & 0 & 1 & 0 & 0 & 0 & CF.01 \\
$\mathbf{6}$ & 0 & 0 & 0 & 1 & 0 & 1 & 2 & CF.01 or CF.03 or CF.06 or CF.09 \\
$\mathbf{7}$ & 0 & 0 & 0 & 1 & 1 & 0 & 2 & CF.01 or CF.04 or CF.07 or CF.08 \\
$\mathbf{8}$ & 0 & 0 & 0 & 1 & 1 & 1 & 3 & CF.01 \\
$\mathbf{9}$ & 0 & 0 & 1 & 0 & 0 & 0 & 1 & CF.1 \\
$\mathbf{1 0}$ & 0 & 0 & 1 & 0 & 0 & 1 & 2 & CF.02 or CF.03 or CF.07 or CF.11 \\
$\mathbf{1 1}$ & 0 & 0 & 1 & 0 & 1 & 0 & 2 & CF.02 or CF.04 or CF.06 or CF.09 \\
$\vdots$ & $\vdots$ & $\vdots$ & $\vdots$ & $\vdots$ & $\vdots$ & $\vdots$ & $\vdots$ & $\vdots$ \\
$\mathbf{6 1}$ & 1 & 1 & 1 & 1 & 0 & 0 & 4 & CF.01 \\
$\mathbf{6 2}$ & 1 & 1 & 1 & 1 & 0 & 1 & 5 & CF.01 \\
$\mathbf{6 3}$ & 1 & 1 & 1 & 1 & 1 & 0 & 5 & CF.01 \\
$\mathbf{6 4}$ & 1 & 1 & 1 & 1 & 1 & 1 & 6 & CF.01
\end{tabular}

4.2. $3 \times 2$ Matrix. A vertical expansion of the $2 \times 2$ matrix presented in Section 4.1 leads to a $3 \times 2$ matrix, that is, three rows and two columns.

The conception of IS is analogous. However, as the number of array modules increases, there are some redundancies on the information system. There are cases in which the clustering only occurs in one of the settings of interest, although, in some situations, there is more than one possible setting.

From (2) and (5), it can be seen that matrix has 64 different possibilities of shading for a six-PV module, which can reach from 0 to 6 modules simultaneously. Among these possibilities, in situations where two or three modules are shaded, array reconfiguration may become feasible and could improve energy extraction. In the opposite case, the system keeps on the reference configuration (CF.01).

According to (3) there are 10 settings of interest, which are presented in Figure 4.

Part of the information system for the $3 \times 2$ array is shown in Table 2. In addition to the attributes A, B, C, D, E, and F, the condition attribute $\mathrm{Q}$ (number of shaded modules) is introduced in the IS to simplify the reducts creation.

From Table 2, it can be seen that $\mathrm{E}$ and $\mathrm{F}$ modules are shaded and the other array modules are illuminated in situation 4, for example. According to the reconfiguration method given in Section 2, modules $\mathrm{E}$ and $\mathrm{F}$ remain grouped in the same series row for the optimal configuration. This situation occurs in the settings of interest $1,2,5$, and 10 as seen in Figure 4.

According to RST, shapes of shade that have more than one configuration of interest, for example, situation 4, are considered as inconsistent. In this case, for the same set of condition attributes, there are different decision attributes. However, in an electric circuit, these situations are redundant, since any setting provides the same result. 

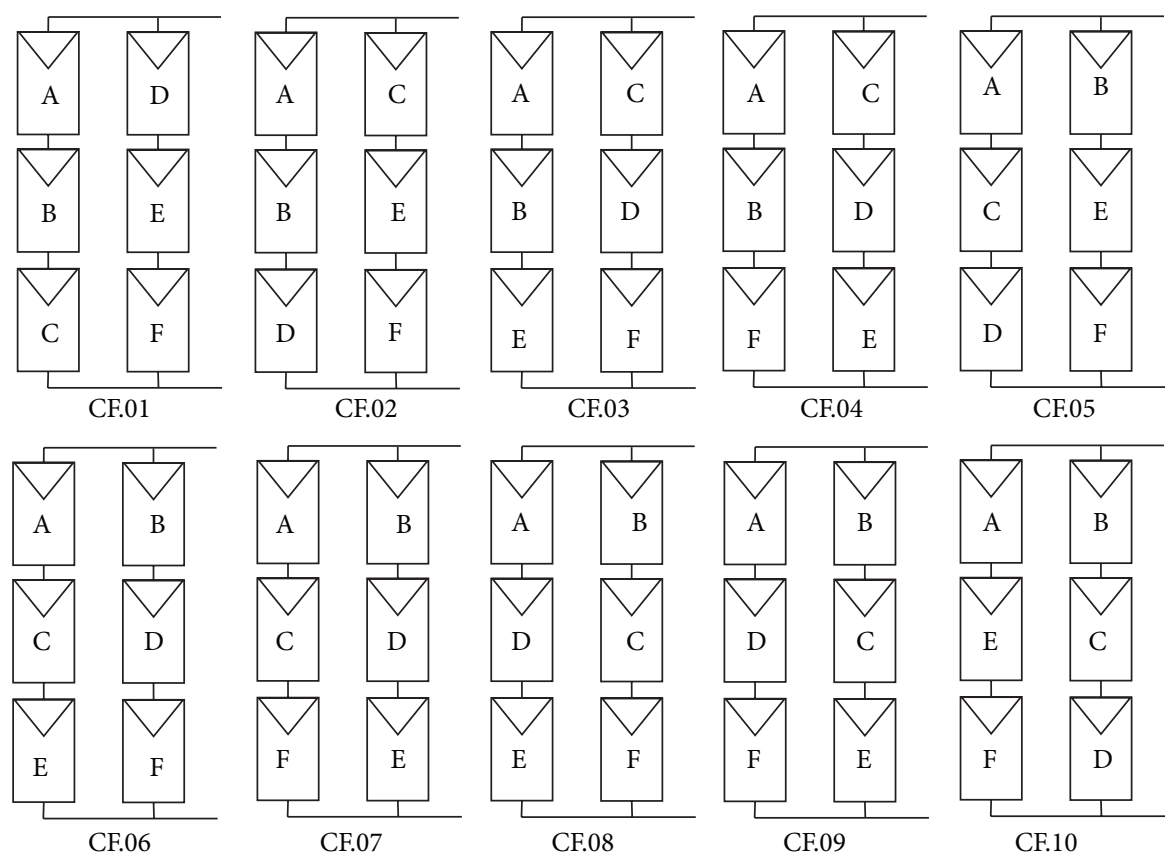

FIGURE 4: Settings of interest for a $3 \times 2$ matrix.

The concept and effectiveness of the knowledge reduction process become evident in the $3 \times 2$ system. From the IS presented in Table 2, it is possible to extract 25 rules that represent the essence of the knowledge base.

Part of 25 rules is written in a simplified form and listed as follows.

(1) $((\mathrm{Q}=0 \mathrm{OR} \mathrm{Q}=1 \mathrm{OR} \mathrm{Q}=4 \mathrm{OR} \mathrm{Q}=5 \mathrm{OR} \mathrm{Q}=6) \mathrm{OR}$ $(\mathrm{D}=1 \mathrm{AND} \mathrm{E}=1 \mathrm{AND} \mathrm{F}=1) \mathrm{OR}(\mathrm{A}=1 \mathrm{AND} \mathrm{B}=1$ AND C = 1) $\rightarrow$ CF.01

(2) $((\mathrm{Q}=3)$ AND $((\mathrm{A}=0$ AND B $=0$ AND D $=0)$ OR $(\mathrm{A}$ $=1$ AND B $=1$ AND D =1)) $\rightarrow$ CF.02

(3) $((\mathrm{Q}=3)$ AND $((\mathrm{A}=0 \mathrm{AND} \mathrm{B}=0$ AND E $=0)$ OR $(\mathrm{A}$ $=1$ AND B $=1$ AND E = 1)) $\rightarrow$ CF.03

(4) $((\mathrm{Q}=3)$ AND $((\mathrm{A}=0$ AND B $=0$ AND F $=0)$ OR $($ A $=1$ AND B $=1$ AND F = 1)) $) \rightarrow$ CF.04

(5) $((\mathrm{Q}=3)$ AND $((\mathrm{A}=0$ AND $\mathrm{C}=0$ AND D $=0)$ OR $(\mathrm{A}=1 \mathrm{AND} \mathrm{C}=1 \mathrm{AND} \mathrm{D}=1))) \rightarrow$ CF.05

(6) $((\mathrm{Q}=3)$ AND $((\mathrm{A}=0$ AND $\mathrm{C}=0$ AND E $=0)$ OR $(\mathrm{A}$ $=1$ AND C $=1$ AND E $=1))) \rightarrow$ CF.06

(7) $((\mathrm{Q}=3)$ AND $((\mathrm{A}=0$ AND $\mathrm{C}=0$ AND F $=0)$ OR $(\mathrm{A}$ $=1$ AND C $=1$ AND F $=1))) \rightarrow$ CF.07

(8) $((\mathrm{Q}=3)$ AND $((\mathrm{A}=0$ AND D $=0$ AND E $=0)$ OR $(\mathrm{A}$ $=1$ AND D $=1$ AND E = 1)) $\rightarrow$ CF.08

(9) $((\mathrm{Q}=3)$ AND $((\mathrm{A}=0$ AND D $=0$ AND F $=0)$ OR $(\mathrm{A}$ $=1$ AND D $=1$ AND F $=1))) \rightarrow$ CF.09

(10) $((\mathrm{Q}=3)$ AND $((\mathrm{A}=0$ AND E $=0$ AND F $=0)$ OR $(\mathrm{A}$ $=1$ AND E $=1$ AND F $=1))) \rightarrow$ CF.10

(11) $(\mathrm{E}=1$ AND $\mathrm{F}=1$ AND $\mathrm{Q}=2) \rightarrow($ CF.01 OR CF.02 OR CF.05 OR CF.10)
(23) $(\mathrm{A}=1 \mathrm{AND} \mathrm{B}=1 \mathrm{AND} \mathrm{Q}=2) \rightarrow($ CF.01 OR CF.02 OR CF.03 OR CF.04)

(24) $(\mathrm{B}=1 \mathrm{AND} F=1 \mathrm{AND} \mathrm{Q}=2) \rightarrow($ CF.04 OR CF.05 OR CF.06 OR CF.08)

(25) $(\mathrm{A}=1 \mathrm{AND} \mathrm{F}=1 \mathrm{AND} \mathrm{Q}=2) \rightarrow($ CF.04 OR CF.07 OR CF.09 OR CF.10)

For each one of the 64 different shading possibilities, only one rule is satisfied, which indicates the most suitable configuration of interest.

In cases where there are redundancies (rules 11 to 25), the choice of the best configuration is performed in a second stage of the algorithm, where the previous configuration is evaluated.

The previous information setting is used to achieve the least number of changes in the layout of modules under shading conditions. The definition of optimal configuration in redundant conditions is made based on two criteria:

(1) if the previous configuration is one of the optimal configurations: the previous setting is maintained;

(2) if the previous configuration is not one of the optimal configurations, it is necessary to choose the setting where the least number of modules is displaced.

4.3. $2 \times 3$ Matrix. The horizontal expansion of the $2 \times 2$ matrix, presented in Section 4.1, leads to a $2 \times 3$ matrix (two rows and three columns).

The procedure for the IS creation and determination of rules is similar to the $3 \times 2$ matrix, presented in Section 4.2. In the $2 \times 3$ system, there are 15 settings of interest as shown in Figure 5. 

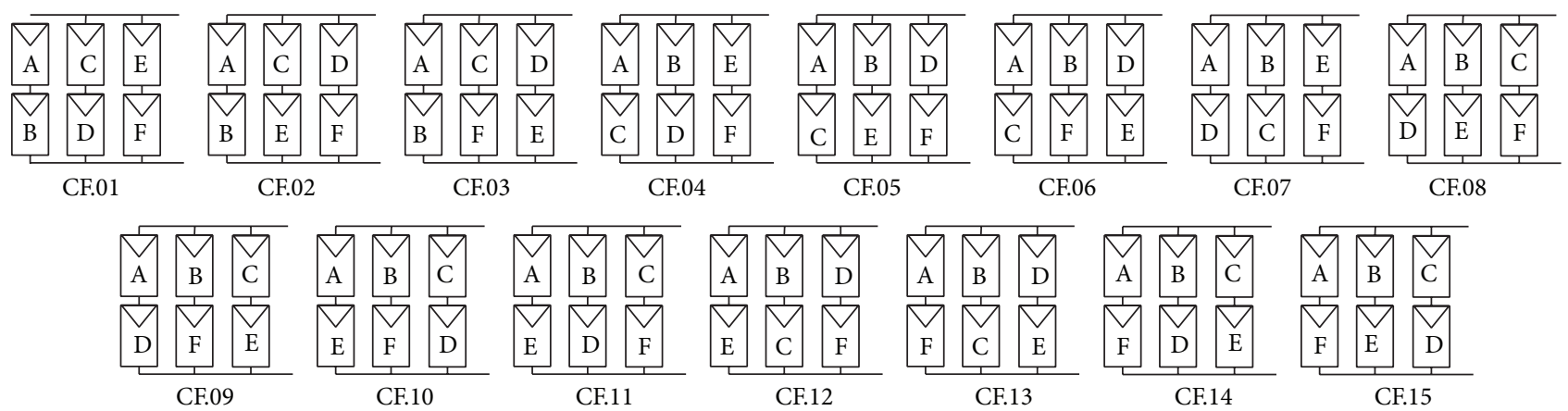

FIGURE 5: Settings of interest for a $2 \times 3$ matrix.

TABLE 3: Part of the Information System of a $2 \times 3$ matrix.

\begin{tabular}{lllllllll}
\hline $\mathrm{U}$ & $\mathrm{A}$ & $\mathrm{B}$ & $\mathrm{C}$ & $\mathrm{D}$ & $\mathrm{E}$ & $\mathrm{F}$ & $\mathrm{Q}$ & $\mathrm{CF}$ \\
\hline $\mathbf{1}$ & 0 & 0 & 0 & 0 & 0 & 0 & 0 & CF.01 \\
$\mathbf{2}$ & 0 & 0 & 0 & 0 & 0 & 1 & 1 & CF.01 \\
$\mathbf{3}$ & 0 & 0 & 0 & 0 & 1 & 0 & 1 & CF.01 \\
$\mathbf{4}$ & 0 & 0 & 0 & 0 & 1 & 1 & 2 & CF.01 or CF.04 or CF.07 \\
$\mathbf{5}$ & 0 & 0 & 0 & 1 & 0 & 0 & 1 & CF.01 \\
$\mathbf{6}$ & 0 & 0 & 0 & 1 & 0 & 1 & 2 & CF.02 or CF.05 or CF.12 \\
$\mathbf{7}$ & 0 & 0 & 0 & 1 & 1 & 0 & 2 & CF.03 or CF.06 or CF.13 \\
$\mathbf{8}$ & 0 & 0 & 0 & 1 & 1 & 1 & 0 & CF.01 or CF.02 or CF.03 or CF.04 or CF.05 or CF.06 or CF.07 or CF.12 or CF.13 \\
$\vdots$ & $\vdots$ & $\vdots$ & $\vdots$ & $\vdots$ & $\vdots$ & $\vdots$ & $\vdots$ & $\vdots$ \\
$\mathbf{5 9}$ & 1 & 1 & 1 & 0 & 1 & 0 & 4 & CF.02 or CF.05 or CF.12 \\
$\mathbf{6 0}$ & 1 & 1 & 1 & 0 & 1 & 1 & 5 & CF.01 \\
$\mathbf{6 1}$ & 1 & 1 & 1 & 1 & 0 & 0 & 4 & CF.01 or CF.04 or CF.07 \\
$\mathbf{6 2}$ & 1 & 1 & 1 & 1 & 0 & 1 & 5 & CF.01 \\
$\mathbf{6 3}$ & 1 & 1 & 1 & 1 & 1 & 0 & 5 & CF.01 \\
$\mathbf{6 4}$ & 1 & 1 & 1 & 1 & 1 & 1 & 6 & CF.01 \\
\hline
\end{tabular}

With the horizontal expansion, the $2 \times 3$ system reconfiguration is feasible in situations where the number of shaded modules is 2, 3, or 4 according to (3). This fact increases the amount of redundant conditions as seen in Table 3.

The rules generated from the IS in Table 3 are similar to those presented in Section 4.2.

(1) $(\mathrm{Q}=0$ OR $\mathrm{Q}=1 \mathrm{OR} \mathrm{Q}=5 \mathrm{OR} \mathrm{Q}=6) \rightarrow($ CF.01 $)$

(2) $((\mathrm{E}=1 \mathrm{AND} F=1 \mathrm{AND} \mathrm{Q}=2) \mathrm{OR}(\mathrm{E}=0 \mathrm{AND} \mathrm{F}=0$ $\mathrm{AND} \mathrm{Q}=4)) \rightarrow($ CF.01 OR CF.04 OR CF.07)

(3) $((\mathrm{A}=0 \mathrm{AND} \mathrm{B}=0 \mathrm{AND} \mathrm{C}=0 \mathrm{AND} \mathrm{Q}=3) \mathrm{OR}(\mathrm{A}=$ $1 \mathrm{AND} B=1 \mathrm{AND} C=1 \mathrm{AND} Q=3)) \rightarrow($ CF.01 OR CF.02 OR CF.03 OR CF.04 OR CF.05 OR CF.06 OR CF.07 OR CF.12 OR CF.13)

(4) $((\mathrm{A}=0 \mathrm{AND} \mathrm{B}=0 \mathrm{AND} \mathrm{D}=0 \mathrm{AND} \mathrm{Q}=3) \mathrm{OR}(\mathrm{A}=$ $1 \mathrm{AND} B=1 \mathrm{AND} D=1 \mathrm{AND} Q=3)) \rightarrow($ CF.01 OR CF.02 OR CF.03 OR CF.04 OR CF.07 OR CF.08 OR CF.09 OR CF.11 OR CF.14)

(5) $((\mathrm{C}=1 \mathrm{AND} \mathrm{D}=1 \mathrm{AND} \mathrm{Q}=2) \mathrm{OR}(\mathrm{C}=0 \mathrm{AND} \mathrm{D}=$ 0 AND $\mathrm{Q}=4)) \rightarrow($ CF.01 OR CF.10 OR CF.15)

(6) $((\mathrm{A}=0 \mathrm{AND} \mathrm{B}=0 \mathrm{AND} \mathrm{E}=0 \mathrm{AND} \mathrm{Q}=3) \mathrm{OR}(\mathrm{A}=$ $1 \mathrm{AND} B=1 \mathrm{AND} E=1 \mathrm{AND} Q=3)) \rightarrow($ CF.01 OR

\section{CF.02 OR CF.03 OR CF.05 OR CF.08 OR CF.10 OR CF.11 OR CF.12 OR CF.15)}

(23) $((\mathrm{B}=1 \mathrm{AND} F=1 \mathrm{AND} \mathrm{Q}=2) \mathrm{OR}(\mathrm{B}=0 \mathrm{AND} \mathrm{F}=0$ AND Q = 4) $\rightarrow($ CF.06 OR CF.09 OR CF.10)

(24) $((\mathrm{B}=1 \mathrm{AND} \mathrm{E}=1 \mathrm{AND} \mathrm{S}=2) \mathrm{OR}(\mathrm{B}=0 \mathrm{AND} \mathrm{E}=0$ AND $S=4)) \rightarrow($ CF.05 OR CF.08 OR CF.15)

(25) $((\mathrm{A}=0 \mathrm{AND} \mathrm{E}=0 \mathrm{AND} \mathrm{Q}=4) \mathrm{OR}(\mathrm{A}=1 \mathrm{AND} \mathrm{E}=$ $1 \mathrm{AND} \mathrm{Q}=2)) \rightarrow($ CF.10 OR CF.11 OR CF.12)

(26) $((\mathrm{A}=0 \mathrm{AND} \mathrm{F}=0 \mathrm{AND} \mathrm{Q}=4) \mathrm{OR}(\mathrm{A}=1 \mathrm{AND} \mathrm{F}=$ $1 \mathrm{AND} \mathrm{Q}=2)) \rightarrow($ CF.13 OR CF.14 OR CF.15)

\section{Simulation Results}

In order to evaluate the proposed methodology, the circuits designed in Section 4 were implemented in PSIM software. The software accurately represents the operation of commercial PV modules and is able to execute microprocessor codes through the use of DLL (Dynamic Link Library) logical block. 


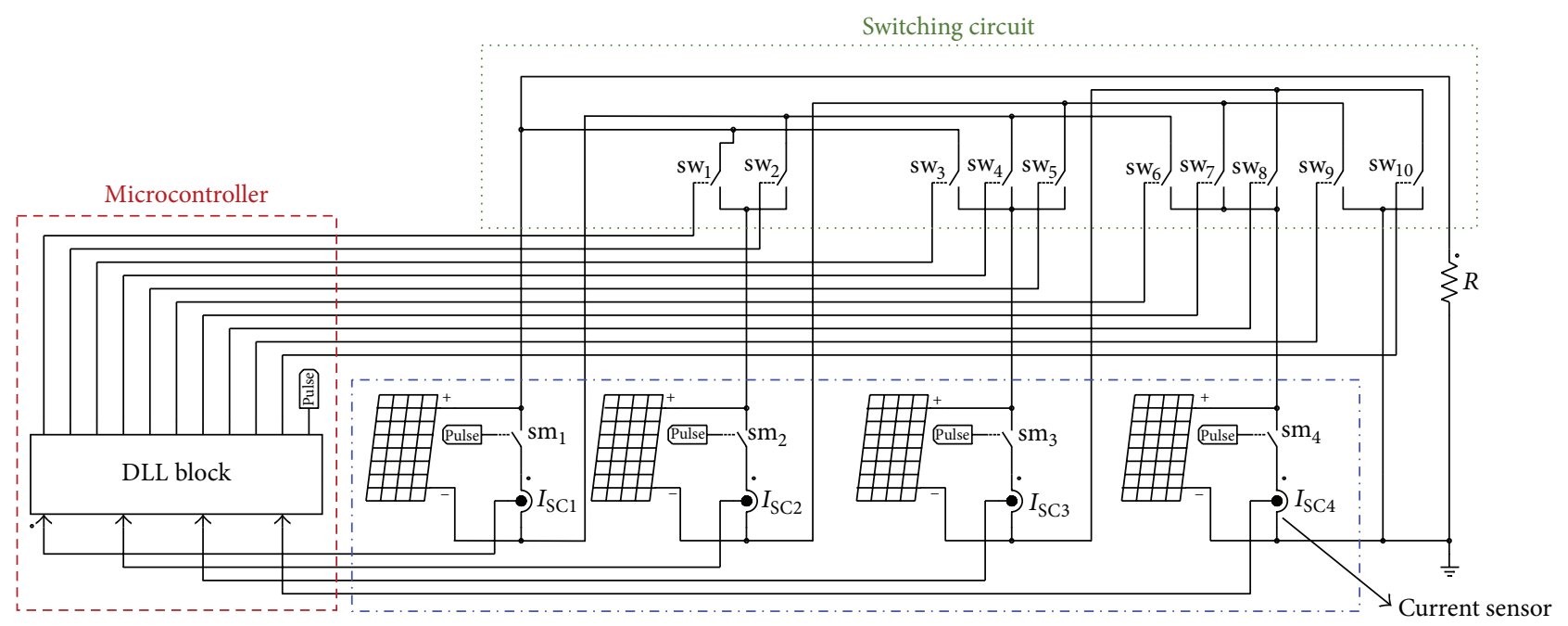

PV array

FIgURE 6: Simplified circuitry of the reconfiguration system for a $2 \times 2$ matrix.

TABLE 4: KS10 specifications.

\begin{tabular}{lc}
\hline Parameters & Value \\
\hline Maximum Power $\left(P_{\mathrm{MPP}}\right)$ & $10 \mathrm{~W}$ \\
Short-circuit current $\left(I_{\mathrm{SC}}\right)$ & $0.62 \mathrm{~A}$ \\
Open-circuit Voltage $\left(V_{\mathrm{OC}}\right)$ & $21.5 \mathrm{~V}$ \\
Maximum Power Current $\left(I_{\mathrm{MPP}}\right)$ & $0.60 \mathrm{~A}$ \\
Maximum Power Voltage $\left(V_{\mathrm{MPP}}\right)$ & $16.9 \mathrm{~V}$ \\
\hline
\end{tabular}

5.1. $2 \times 2$ Matrix. Figure 6 shows the simplified circuitry of the reconfiguration system for a $2 \times 2$ matrix. The circuit can be divided into three individual blocks: PV array, microcontroller, and switching circuit.

The array consists of four PV modules that simulates the behavior of the KS10 by Kyocera module. Its specifications are presented in Table 4.

Since the output current through each module is proportional to the respective solar irradiance, shading is identified by periodic measurements of the short-circuit current $I_{\mathrm{SC}}$. The measurements of currents $I_{\mathrm{SC}}$ are synchronized with the alterations of irradiance in the photovoltaic modules. At each new condition of irradiance, the switches $\mathrm{sw}_{1}, \mathrm{sw}_{2}, \ldots, \mathrm{sw}_{N}$ are opened and the short-circuit currents $I_{\mathrm{SC} 1}, I_{\mathrm{SC} 2}, \ldots, I_{\mathrm{SCN}}$ are measured through the switching modules $\mathrm{sm}_{1}, \mathrm{sm}_{2}, \ldots, \mathrm{sm}_{N}$.

The DLL block executes an algorithm compiled with DEV C++ software. This algorithm is built from the rules obtained with ROSETTA program as presented in Section 4. The flowchart of the proposed method is shown in Figure 7.

In the flowchart, the current supplied by each photovoltaic array module is read $\left(I_{\mathrm{SC}}(k)\right)$. If this current is lower than the reference current $\left(I_{\text {ref }}\right)$, the module is considered shaded. Otherwise, the module is considered unshaded. The information of each module is sent to the DLL block, where the corresponding rule is chosen. After the definition of the suitable configuration, the switching circuit is set.

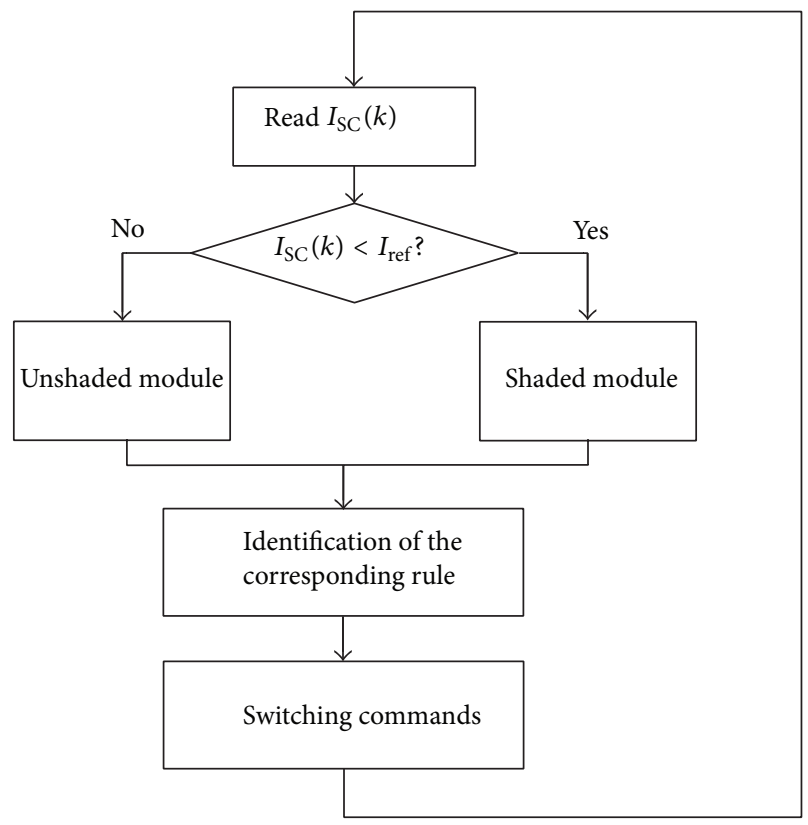

FIGURE 7: Flowchart of the proposed method.

PV systems are usually associated with MPPT systems [13]. However, in order to properly evaluate if the reconfiguration provides improved results, the module was connected to a resistive load. The resistance value has been established for the system to operate close to the maximum power point (MPP) under standard test conditions (STC-1000 W/m ${ }^{2}$ and $25^{\circ} \mathrm{C}$ ). Therefore, when shading occurs, the operating conditions change and the system no longer operates at the MPP.

To evaluate the sixteen different shading situations in a single simulation test, the current through each PV module is adjusted. In the following tests, the module current has been reduced by $40 \%$ so that shading situations can be emulated. 

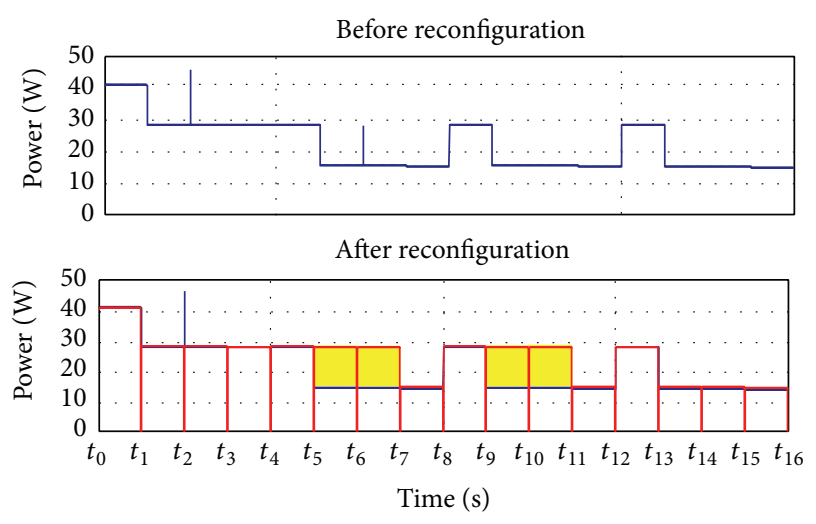

FIGURE 8: Power versus time curve for a $2 \times 2$ array and progressive shading (before and after reconfiguration).

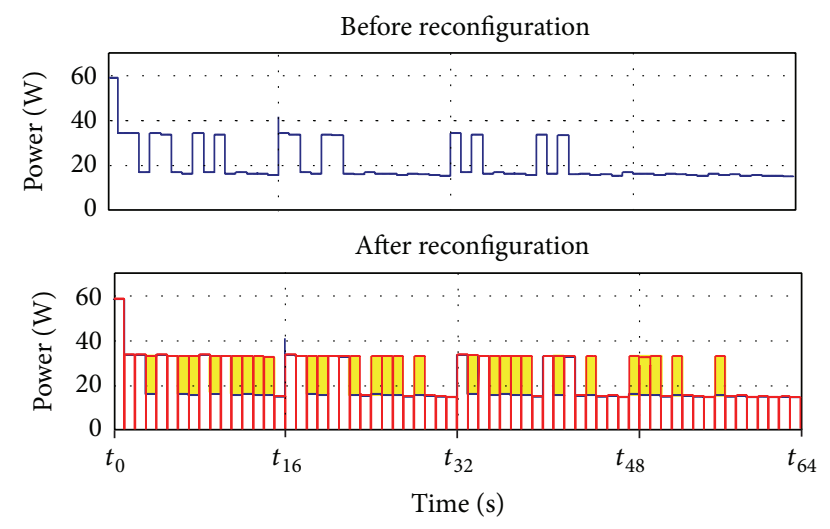

FIGURE 9: Power versus time curve for a $3 \times 2$ array and progressive shading (before and after reconfiguration).

The curves presented in Figure 8 reproduce the output power of PV array, in all shading situations, before and after reconfiguration.

The shading sequence is performed according to Table 1. In the first interval $\left(t_{0}-t_{1}\right)$, all modules are unshaded. In the second one $\left(t_{1}-t_{2}\right)$, module D is shaded, and so on. Among 16 different shading possibilities, reconfiguration is feasible in only four situations. Under the system reconfiguration, it is possible to increase the power output from $15 \mathrm{~W}$ to $29 \mathrm{~W}$, which corresponds to an increase about $14 \mathrm{~W}$.

5.2. $3 \times 2$ Matrix. The reconfiguration system developed for a $3 \times 2$ array is similar to the one presented in the previous section. Six KS10 PV modules are connected to the switching circuit and control system. The module is connected to a fixed resistive load, and the power output is sampled before and after the system reconfiguration.

Figure 9 presents the power output curves before and after system reconfiguration for 64 different shading situations. The increase of output power after system reconfiguration changed from $17 \mathrm{~W}$ to $34 \mathrm{~W}$, that is achieved with the reconfiguration of electrical connections.

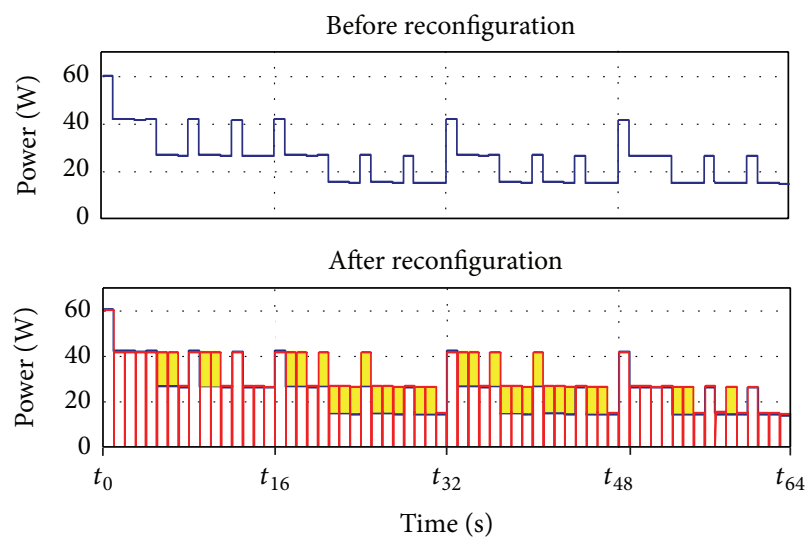

FIGURE 10: Power versus time curve for a $2 \times 3$ array and progressive shading (before and after reconfiguration).

5.3. $2 \times 3$ Matrix. The simulation results for the $2 \times 3$ matrix are shown in Figure 10. It also confirms the effectiveness of the proposed method.

Although the $2 \times 3$ system has the same number of modules as the $3 \times 2$ system, it is important to notice that the results (of the $3 \times 2$ matrix) are quite distinct. This new arrangement has one extra column. Its reconfiguration system seems to be more robust, since the reconfiguration is possible even in situations with four shaded modules, which does not occur in the $3 \times 2$ system.

The simulation results for 64 shading situations are seen in Figure 10. There are different power levels due to the physical structure of the arrangement, which enhance the possible situations of reconfigurations but limit the concentration of modules in a smaller number of columns.

For example, the output power is reduced in the $3 \times$ 2 arrangement in the case of three shaded PV modules, what can be mitigated with the concentration of all affected modules in one series row. In the $2 \times 3$ arrangement, the shading of three PV modules inevitably reaches two columns of the matrix even after the system reconfiguration, thus reducing the output power.

\section{Experimental Results}

In order to evaluate the proposed method, a reconfiguration system with four PV modules $(2 \times 2)$ was implemented. The system is presented in Figure 11.

The implementation of this reconfiguration methodology in photovoltaic systems requires additional circuitry such as sensors, switching, and the control system (hardware and software). Those structures can be seen in the schematic of Figure 6.

The PV array is composed of four KS10 modules (Table 4) resulting in a total output power of $40 \mathrm{~W}$. The identification of each module condition is made through the measurement circuit.

The shading identification is performed by measuring the short-circuit current $I_{\mathrm{SC}}$. The measurements are performed 


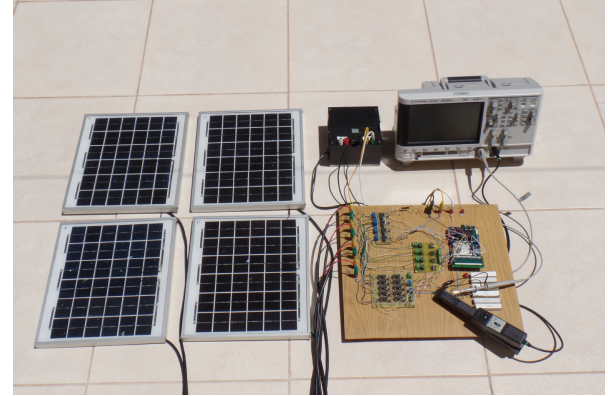

FIGURE 11: Reconfiguration system.

on 10-second interval $(f=0.1 \mathrm{~Hz})$ and the current of each PV module is monitored through an INA193 current sensor.

After signal conditioning, the parameter specifications are sent to the microprocessor (PSoC-CY8C29466). Then, the switching circuit, which is composed of reed relays (HE721A1210), is triggered.

Partial shading conditions were created with the use of tracing paper, which produce uniform shading, thus filtering the incident radiation [8]. Although the shading profiles that affect the PV array in the real system are different from the pattern created by the paper, its use is justified by the necessity of controlling the shading intensity. In the performed tests, the tracing paper has presented a uniform shade between $40 \%$ and $45 \%$.

The PV array is connected to a fixed resistive load, where voltage, current, and output power are measured. During the tests, the module temperature reached $60^{\circ} \mathrm{C}$, which consequently reduces the available power.

As discussed in Section 5.1, the $2 \times 2$ matrix has four shading conditions where the system reconfiguration is interesting. Thus, the following sections present the experimental results of the aforementioned case studies.

6.1. Two Shaded Modules ( $A$ and $C$ ): CF.01 $\rightarrow$ CF.02. Figure 12 presents the waveforms for the current, voltage, and output power. The time interval includes the shading of modules $\mathrm{A}$ and $\mathrm{C}$ and also the performance of the reconfiguration system.

The test shown in Figure 12 lasts 10 seconds and can be divided into four time intervals.

$t_{0}$ to $t_{1}$-all modules are illuminated and remain in configuration 1 .

$t_{1}$ to $t_{2}-\mathrm{A}$ and $\mathrm{C}$ modules are shaded; the transient oscillation due to artificial shading can also be noticed.

$t_{2}$ to $t_{3}$-measurement time; all matrix switches are off in order to measure the modules' parameters.

$t_{3}$ to $t_{4}$-reconfigured system: configuration 2 .

When modules A and C are shaded, the setting of interest is configuration 2. Through reconfiguration, it is possible to increase the output power by about $13.5 \mathrm{~W}$ according to Figure 12.

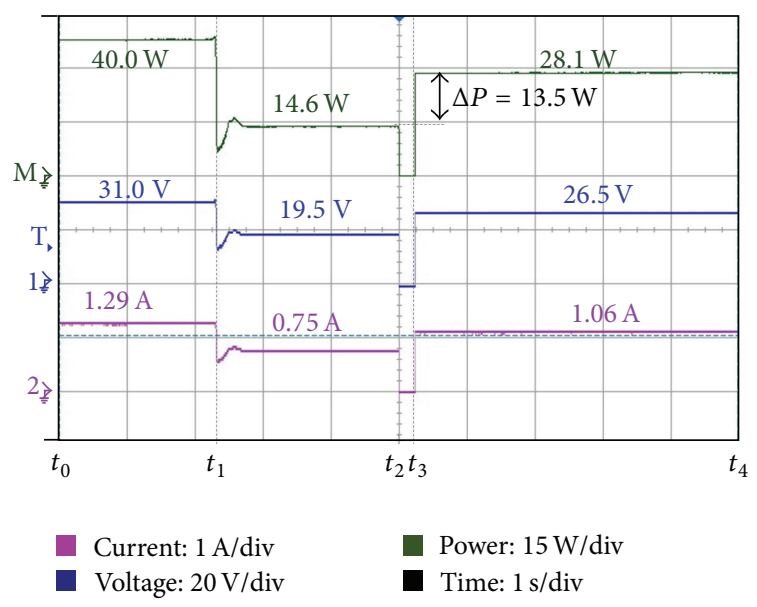

FIgURE 12: Experimental results: CF.01-CF.02.

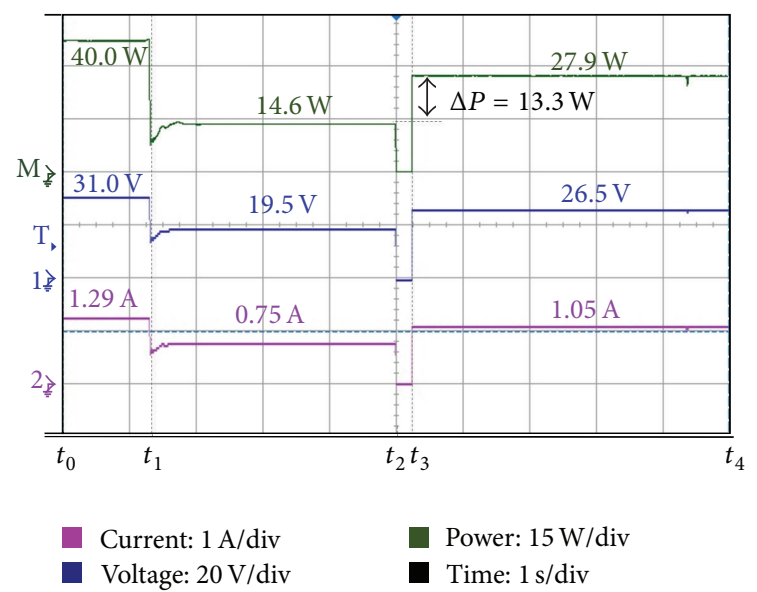

FIGURE 13: Experimental results: CF.01-CF.03.

6.2. Two Shaded Modules (B and C): CF.01 $\rightarrow$ CF.03. A similar test is performed to verify the system performance when modules B and C are shaded. As expected, the output power increases by $13.3 \mathrm{~W}$ after system reconfiguration as seen in Figure 13.

6.3. Two Shaded Modules $(A$ and $C) \rightarrow(A$ and $D): C F .02 \rightarrow$ CF.03. Analogous to the previous sections, this test can also be divided into four time intervals as seen in Figure 14. However, modules $\mathrm{A}$ and $\mathrm{C}$ in this test are shaded in the time interval between $t_{0}$ and $t_{1}$, and the setting of interest is configuration 2. Between $t_{1}$ and $t_{2}$, the shading profile changes, while modules $\mathrm{A}$ and $\mathrm{D}$ are now shaded, thus causing the output power to be reduced.

After the measurement period ( $t_{2}$ to $\left.t_{3}\right)$, the reconfiguration system chooses the new setting of interest as configuration 3 ( $t_{3}$ to $\left.t_{4}\right)$.

6.4. One Shaded Module (A): CF.01 $\rightarrow$ CF.01. As indicated in Section 2, there are some shading conditions where nothing can be done. The test carried out in Figure 15 evidences this situation, where a single module is shaded. 


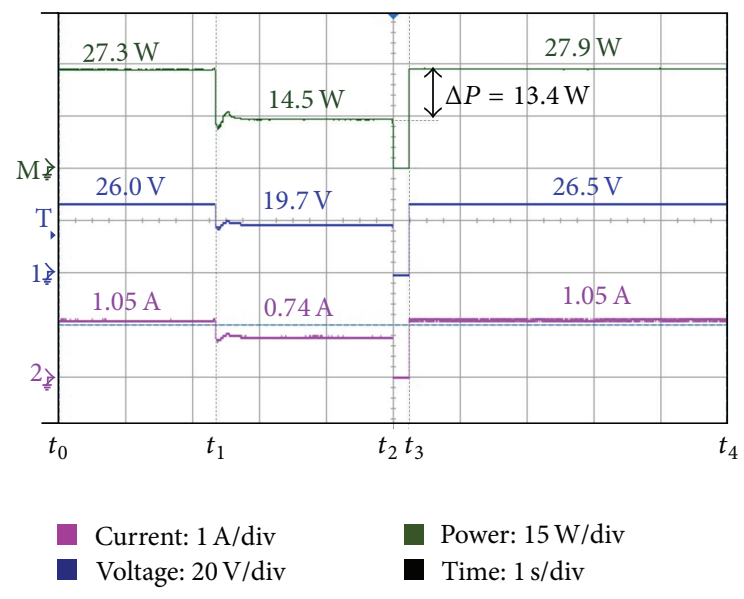

FIGURE 14: Experimental results: CF.02-CF.03.

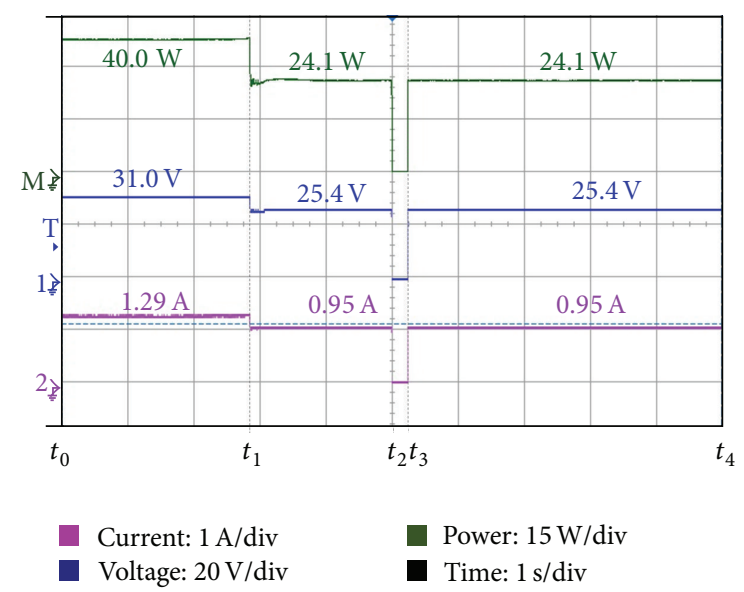

FIgURE 15: Experimental results: CF.01-CF.01.

At the beginning, all modules are illuminated and, after $t_{1}$, module $\mathrm{A}$ is shaded. Then, the system parameters are measured, and the setting of interest keeps the same configuration (configuration 1). As expected, there is no change in the output power.

\section{Conclusions}

This paper has presented the design of a dynamic reconfiguration system for shaded PV arrays with SP interconnection. The proposed method is characterized by the maintenance of the PV array size; that is, the array dimensions do not change.

The methodology is based in Rough Sets Theory, in which the behavior of the proposed reconfiguration system can be defined by simple logical rules, implemented in a low-coast microcontroller.

The operation of the proposed system has been evaluated through computer simulations and experimental tests, while the obtained results have shown its effectiveness. When compared to an array with static connections, the reconfiguration system provides improved extraction of energy under partial shading conditions.
Thus, reconfiguration provides a significant increase in the output power under different situations. The negative effects of shading in the overall efficiency of the PV system are minimized by the reorganization of the array electrical connections; that is, the shaded PV modules are reconnected in the best possible position, according to the settings of interest.

\section{Conflict of Interests}

The authors declare that there is no conflict of interests regarding the publication of this paper.

\section{Acknowledgments}

The authors would like to thank CAPES, CNPq, and FAPEMIG for the financial support for this work.

\section{References}

[1] H. S. Rauschenbach, "Electrical output of shadowed solar arrays," IEEE Transactions on Electron Devices, vol. 18, no. 8, pp. 483-490, 1971.

[2] A. Abete, E. Barbisio, F. Cane, and P. Demartini, "Analysis of photovoltaic modules with protection diodes in presence of mismatching," in Proceedings of the 21st IEEE Photovoltaic Specialists Conference, vol. 2, pp. 1005-1010, May 1990.

[3] J. H. R. Enslin, M. S. Wolf, D. B. Snyman, and W. Swiegers, "Integrated photovoltaic maximum power point tracking converter," IEEE Transactions on Industrial Electronics, vol. 44, no. 6, pp. 769-773, 1997.

[4] B. Patnaik, P. Sharma, E. Trimurthulu, S. P. Duttagupta, and V. Agarwal, "Reconfiguration strategy for optimization of solar photovoltaic array under non-uniform illumination conditions," in Proceedings of the 37th IEEE Photovoltaic Specialists Conference (PVSC '11), pp. 1859-1864, June 2011.

[5] B. Patnaik, J. Mohod, and S. P. Duttagupta, "Dynamic loss comparison between Fixed-State and Reconfigurable Solar Photovoltaic Array," in Proceedings of the 38th IEEE Photovoltaic Specialists Conference (PVSC '12), pp. 1633-1638, June 2012.

[6] M. Alahmad, M. A. Chaaban, S. K. Lau, J. Shi, and J. Neal, "An adaptive utility interactive photovoltaic system based on a flexible switch matrix to optimize performance in real-time," Solar Energy, vol. 86, no. 3, pp. 951-963, 2012.

[7] J. Storey, P. R. Wilson, and D. Bagnall, "The optimized-string dynamic photovoltaic array," IEEE Transactions on Power Electronics, vol. 29, no. 4, pp. 1768-1776, 2014.

[8] P. dos Santos, E. M. Vicente, and E. R. Ribeiro, "Relationship between the shading position and the output power of a photovoltaic panel," in Proceedings of the Brazilian Power Electronics Conference (COBEP '11), pp. 676-681, September 2011.

[9] J. D. Bastidas-Rodriguez, C. A. Ramos-Paja, and A. J. SaavedraMontes, "Reconfiguration analysis of photovoltaic arrays based on parameters estimation," Simulation Modelling Practice and Theory, vol. 35, pp. 50-68, 2013.

[10] Z. Pawlak, Rough Sets: Theoretical Aspects of Reasoning about Data, Kluwer Academic, London, UK, 1991.

[11] J. Komorowski, Z. Pawlak, L. Polkowski, and A. Skowron, "Rough sets: a tutorial," in Rough Fuzzy Hybridization: A New Trend in Decision-Making, S. K. Pale and A. Skowron, Eds., Springer, Singapore, 1999. 
[12] A. Øhrn and J. Komorowski, "ROSETTA: a rough set toolkit for analysis of data," in Proceedings of the 3rd International Joint Conference on Information Sciences (JCIS '97), Aachen, Germany, September 1997.

[13] K. S. Tey, S. Mekhilef, H.-T. Yang, and M.-K. Chuang, "A differential evolution based MPPT method for photovoltaic modules under partial shading conditions," International Journal of Photoenergy, vol. 2014, Article ID 945906, 10 pages, 2014. 

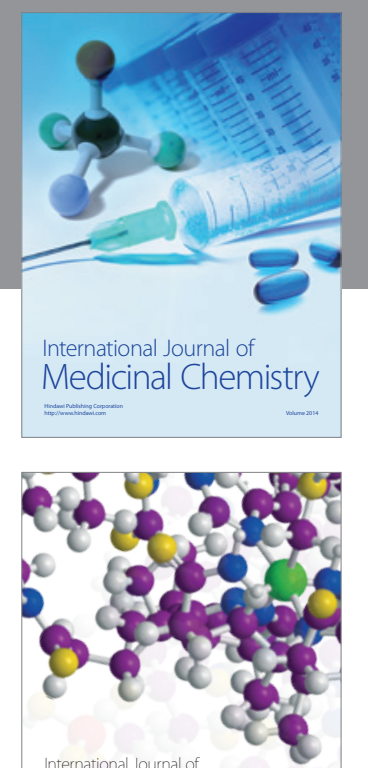

\section{Carbohydrate} Chemistry

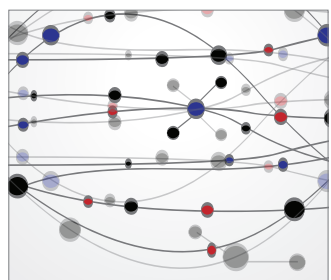

The Scientific World Journal
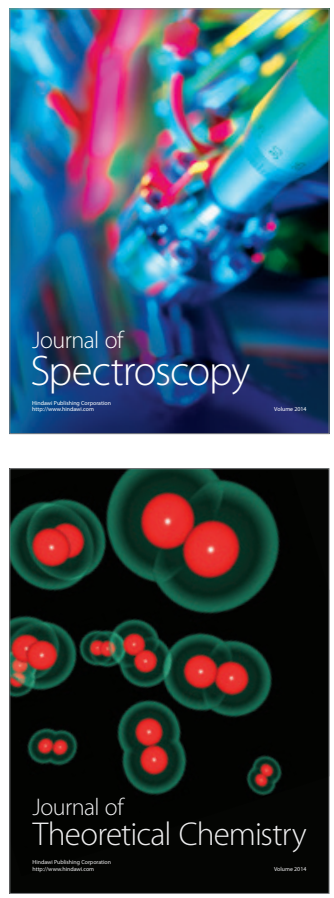
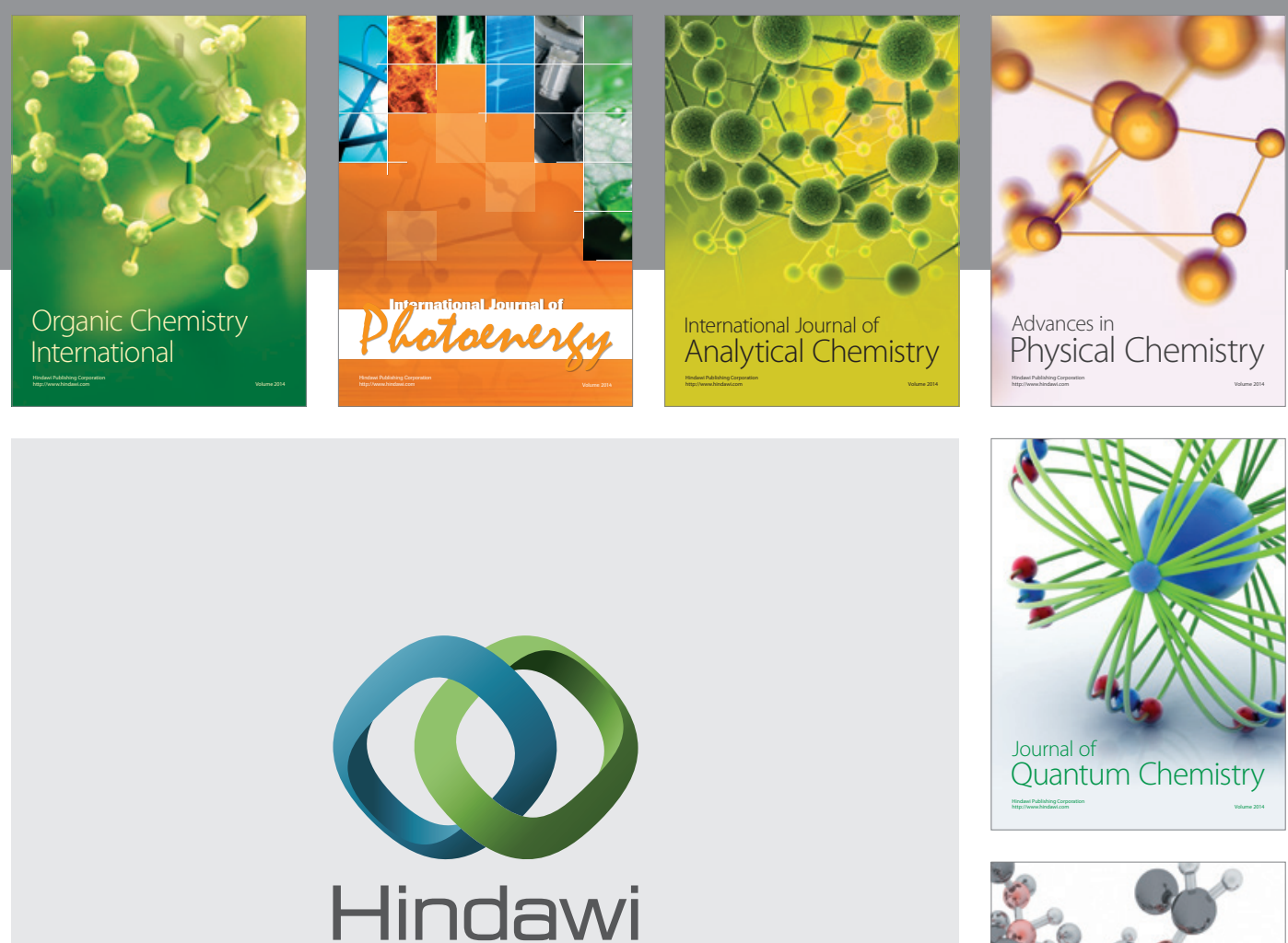

Submit your manuscripts at

http://www.hindawi.com

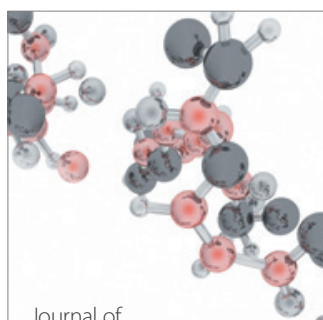

Analytical Methods

in Chemistry

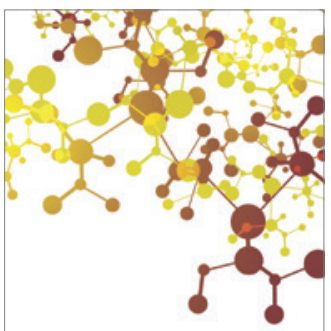

Journal of

Applied Chemistry

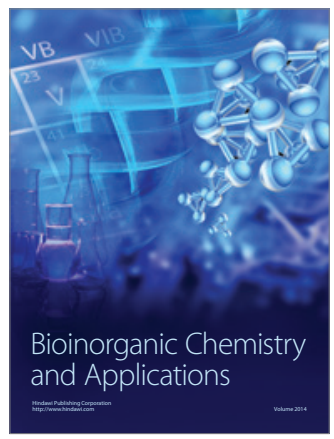

Inorganic Chemistry
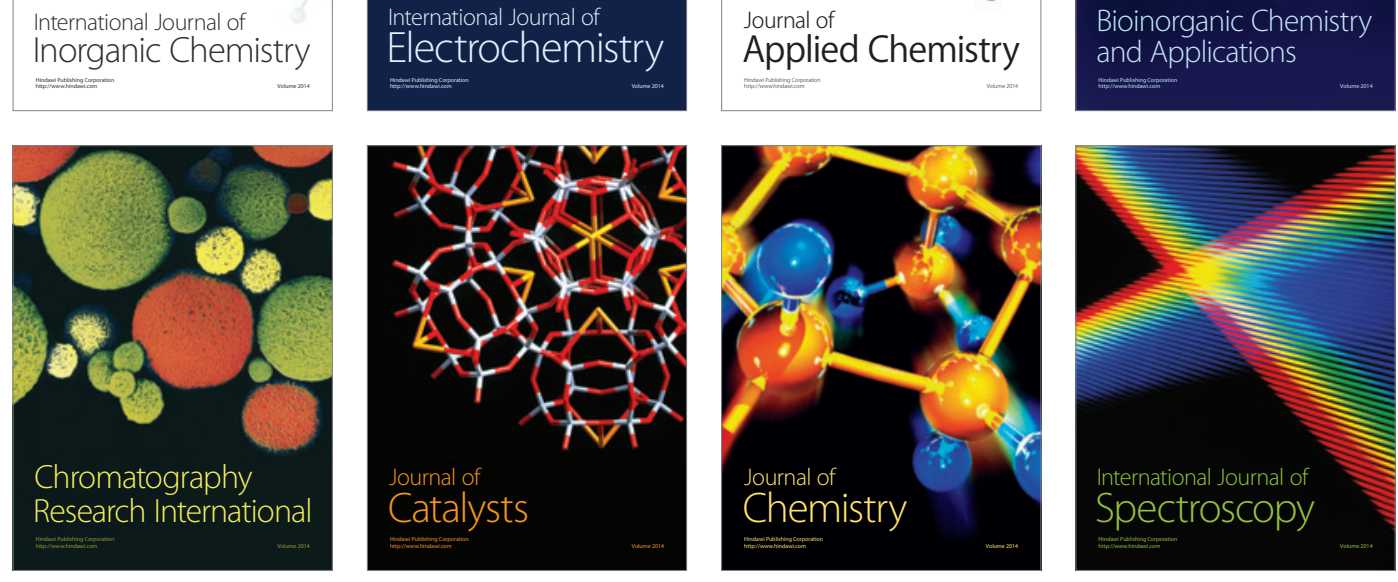